\title{
The Effect of Ion Size on Rate of Dissociation: RRKM Calculations on Model Large Polypeptide Ions
}

\author{
Lawrence L. Griffin \\ Department of Marine Sciences, Texas A \& M University at Galveston, Galveston, Texas, USA \\ David J. McAdoo \\ Marine Biomedical Institute, University of Texas Medical Branch, Galvestan, Texas, USA
}

The larger an ion is, the less likely it is to decompose on mass spectrometry time scales at given critical and internal energies. This is an obstacle to obtaining structural information on large molecules by mass spectrometry. We performed RRKM calculations on model ions with masses from $0.27 \mathrm{kDa}$ to $102.4 \mathrm{kDa}$ to explore what such calculations predict regarding this limitation. According to the calculations, it is impractical to add enough energy to fragment very large ions unless the decomposition has a low critical energy. It is suggested that ion-molecule reactions that are either very low in their critical energies or exothermic may be a feasible approach to fragmenting ionized macromolecules. (I Am Soc Mass Spectrom 1993, 4, 11-15)

$\mathrm{T}$ The use of mass spectrometry to characterize macromolecules is growing dramatically following the development of a variety of techniques [1-4] for generating large ions in the gas phase. However, structural information provided by mass spectrometry on large molecules is limited because the latter do not readily fragment $[5,6]$. Statistical rate theories suggest that fragmentation slows as ions get larger because their internal energy gets distributed over an increasing number of degrees of freedom, and is less frequently sufficiently concentrated in the reaction coordinate to produce fragmentation. To consider strategies for circumventing the effect of increasing ion size on fragmentation rates, it would be useful to know more precisely the predictions of theory regarding the decomposition rates of large, ionized biopolymers. However, it should be noted that in one recent study [7], it was suggested that statistical theories may not apply without modification to the dissociations of large ions.

Recently McKeown and Johnston [8] used the Rice-Rampsberger-Kassel (RRK) expression

$$
k=\nu\left(\frac{E-E_{0}}{E}\right)^{N-1}
$$

Address reprint requests to Lawrence L. Griffin, Department of Marine Sciences, Texas A \& M University at Galveston, Galveston, TX 77553. to investigate large reaction systems, where $\nu$ is the frequency factor, $E$ is the internal energy, and $E_{0}$ is the critical energy for the reaction. They obtained a rate constant $k=10^{-45} \mathrm{~s}^{-1}$ at an $E_{0}$ of $48 \mathrm{~kJ} \mathrm{~mol}^{-1}$ and $E=386 \mathrm{~kJ} \mathrm{~mol}^{-1}$ in a system with $N=1000$ degrees of freedom. Huwever, when they equated ion internal energy with the thermal energy content, which increases in proportion to the size of the reactant, their calculated rates actually increased slightly with ion size. Experimentally, they found that ratios of metastable peak abundances to the intensities of product ions formed in the ion source by the same process were less dependent on precursor size than predicted by simple RRK theory. They concluded that fragmentation rates were determined by contributions of both thermal and added energy.

The RRK theory is the classical approximation of the Rice-Rampsberger-Kassel-Marcus (RRKM) theory. According to the latter, the rate constant $k(E)$ at a given energy is

$$
k(E)=\frac{\sigma}{h} \frac{G^{*}\left(E-E_{0}\right)}{\rho(E)}
$$

where $G^{*}\left(E-E_{0}\right)$ is the sum of the number of states in the transition state at $E-E_{0}, \rho(E)$ is the density of states in the reactant ion at energy $E_{r} \sigma$ is the reaction path degeneracy, and $h$ is Planck's constant. Early detailed RRKM calculations and experiment [9-11] indicated that, as precursor ions become larger, on 
average the fraction of available energy deposited in a product ion becomes smaller, making it less reactive, even when thermal energy was included in the energy content of the ion.

The RRK expression does not reliably make quantitative predictions, as $\nu$ is usually treated as an adjustable parameter [12]. Furthermore, RRKM calculations of fragmentation rates with increasing ion size using expression 2 do not to our knowledge exist for very high molecular weight ions. Therefore, we have performed such calculations on a cleavage in a homologous series of model ionized peptides to determine the theoretical effect of increasing ion size on fragmentation rates and to consider strategies for circumventing the effect of increasing ion size on rates of decomposition. Some of our results have been presented in preliminary form [13].

\section{RRKM Calculations}

For our study, we considered cleavage of the C-terminal peptide bond over a family of model ions simulating polymers of 2-800 alanine-glycine (AG) dipeptide units (105-40,803 degrees of freedom). A slightly modified version of the RRKM program of Hase and Bunker [14] was employed to calculate rate constants using semiclassical (Whitten-Rabinovitch) state counting. For state counting, we utilized models assuming two different sets of vibrational frequencies. Both sets of frequencies neglected side-chain interaction and $\mathrm{H}$-bonding along the peptide backbone, and only the second allows for cooperative modes. Our first model is analogous to an $n$-unit linear polymer with degenerate frequencies [15]. Since ionized polypeptides probably have $\alpha$-helical regions and globular structures, our frequencies probably do not precisely approximate the frequencies of ionized peptides.

For our first model, we assumed that the normal modes of a molecular ion or transition state are reasonably approximated by the set of all individual bond stretches plus bending modes assuciated with individual structural units (e.g., $\mathrm{CH}_{2}, \mathrm{CO}$, etc.) [16] in each polypeptide residue. The frequencies for each mode of the alanylglycine ion (AG-1) and for $N$-alanyl and $C$-glycyl of AG- $n$ were selected from values associated with analogous regions of small molecules and ions $[17,18]$. For AG- $n$, this model incorporated one set of 54 AG frequencies (Table 1) and $n-1$ degenerate sets of GA (glycyl-alanyl) frequencies chosen in a similar manner (Table 2). Transition state frequencies were approximated for each ion by altering $A G$ frequencies associated with the atoms of the first $A-G$ peptide bond, selecting the $\mathrm{CN}$ stretch as the reaction coordinate (Table 3 ), and adding the $n-1 \mathrm{GA}$ stretches and bends of the reactant ion. We compared results of RRKM calculations for AG-1 using our estimated frequencies (Tables 1 and 2) with results from a set adapted from scaled ab initio 3-21G frequencies for the equilibrium structure of the unionized alanylglycine
Table 1. Estimated frequencies $\left(\mathrm{cm}^{-1}\right)$ for alanylglycine ${ }^{+}$.

\begin{tabular}{rrrrrrr}
\hline \hline 85 & 100 & 135 & 135 & 270 & 270 & 400 \\
405 & 410 & 415 & 430 & 430 & 500 & 605 \\
605 & 640 & 640 & 750 & 780 & 800 & 845 \\
845 & 850 & 950 & 1020 & 1045 & 1100 & 1160 \\
1180 & 1200 & 1200 & 1265 & 1280 & 1300 & 1340 \\
1350 & 1420 & 1460 & 1460 & 1460 & 1480 & 1625 \\
1770 & 1790 & 3000 & 3010 & 3010 & 3030 & 3030 \\
3030 & 3300 & 3400 & 3400 & 3585 & & \\
\hline
\end{tabular}

(A. Lowrey, personal communication). With $E_{0}=100$ $\mathrm{kJ} \mathrm{mol}{ }^{-1}$, at a given internal energy rates from RRKM calculations using the $a b$ initio frequencies were about $10 \%$ lower than rates using the estimated frequencies in our first model.

To determine the effects of neglecting lower frequency cooperative modes in the original model, we devised a second set of model ions (LV). Frequencies for the $\mathrm{LV}$ model reactants and transition states were also formally associated with $A$ and $G$ (54 modes) or with interior GAs (51 modes each). For the GA in $A G-2$, we reduced frequencies of the original GA modes as follows: no corrections for $\nu>2000 \mathrm{~cm}^{-1}$, and reductions that ranged from $0.5 \%$ for $2000 \mathrm{~cm}^{-1}$ $z \nu>1000 \mathrm{~cm}^{-1}$ to $0.1 \%$ fur the range $300 \mathrm{~cm}^{-1} \geq \nu$ $\geq 25 \mathrm{~cm}^{-1}$. With the addition of each GA unit, the same factors were applied sequentially to the frequencies for the preceding polymer from the outermost to the innermost GA. Thus for GAs 1 and $n-1$, corrections were applied once, while for the innermost GA units, the corrections were applied $(n-1) / 2$ times. Therefore, as LV model ions increased in size, they were assigned vibrations of decreasing frequencies and increasing numbers of low frequency vibrations (Table 4).

For each model, we used our frequencies to compute each ion's ensemble average vibrational energy at $25{ }^{\circ} \mathrm{C}$ based on the expression [19]

$$
\langle E\rangle=R T^{2} \frac{\partial \ln q}{\partial T}=\sum_{i} \frac{h \nu_{i}}{2}+\sum_{i} \frac{h \nu_{i}}{\exp \left(h \nu_{i} / k T\right)-1}
$$

where $q$ is the vibrational partition function. The summations are over all modes. The thermal energy $\left(E_{\mathrm{T}}\right)$

Table 2. Frequencies $\left(\mathrm{cm}^{-1}\right)$ added to expand a peptide by one Gly-Ala unit

\begin{tabular}{rrrrrrr}
\hline \hline 100 & 135 & 135 & 270 & 270 & 400 & 405 \\
410 & 415 & 430 & 430 & 430 & 500 & 605 \\
605 & 640 & 640 & 750 & 800 & 800 & 845 \\
845 & 850 & 950 & 950 & 1020 & 1045 & 1100 \\
1100 & 1160 & 1200 & 1200 & 1200 & 1280 & 1300 \\
1340 & 1350 & 1460 & 1460 & 1460 & 1480 & 1770 \\
1770 & 3000 & 3010 & 3010 & 3030 & 3030 & 3030 \\
3300 & 3300 & & & & & \\
\hline
\end{tabular}


Table 3. Estimated transition state frequencies $\left(\mathrm{cm}^{-1}\right)$ for Ala-Gly

\begin{tabular}{rrrrrrr}
\hline \hline 100 & 135 & 135 & 185 & 200 & 270 & 270 \\
400 & 400 & 405 & 410 & 415 & 430 & 500 \\
500 & 605 & 640 & 700 & 750 & 780 & 845 \\
845 & 850 & 900 & 1045 & 1100 & 1100 & 1160 \\
1180 & 1200 & 1265 & 1280 & 1300 & 1340 & 1350 \\
1420 & 1460 & 1460 & 1460 & 1480 & 1625 & 1770 \\
1790 & 3000 & 3010 & 3010 & 3030 & 3030 & 3030 \\
3300 & 3400 & 3400 & 3585 & & & \\
\hline
\end{tabular}

was computed as the second sum in eq $3 . E_{\mathrm{T}}$ is about $17.6 \mathrm{~kJ} \mathrm{~mol}^{-1}$ per $\mathrm{AG}$ at $25^{\circ} \mathrm{C}$ in our first model. A practical amount of energy that can be added to an ion in the gas phase is that of a $193-\mathrm{nm}$ photon $(620 \mathrm{~kJ}$ $\mathrm{mol}^{-1}$ ) [20]. Therefore, RRKM rates for the largest ions were calculated at energies from $200 \mathrm{~kJ} \mathrm{~mol}^{-1}$ to above the $E_{T}$ of the system plus the energy of a 193-rum photon.

Rate constants were calculated using the first set of model frequencies for a series of ions from $A G-2$ to $A G-800$ as a function of ion energy at $E_{0}=9.65 \mathrm{~kJ}$ $\mathrm{mol}^{-1}\left(0.1 \mathrm{eV}\right.$ ) (Figure 1) and at $E_{0}=100 \mathrm{~kJ} \mathrm{~mol}-1$ (Figure 2). Calculated rate constants at each $E$ shifted to smaller values with increasing size of the ion. The energy corresponding to $E_{\mathrm{T}}+193 \mathrm{~nm}$ was determined and marked on each curve. For $E_{0}=9.65 \mathrm{~kJ} \mathrm{~mol}^{-1}$, rate constants for the original model ions were all above $10^{10} \mathrm{~s}^{-1}$ at this point, indicating average ion lifetimes less than $10^{-10} \mathrm{~s}$ for even the largest ions.

For $E_{0}=100 \mathrm{~kJ} \mathrm{~mol}^{-1}$, average lifetimes calculated at $E_{\mathrm{T}}+193 \mathrm{~nm}$ varied from $2.7 \times 10^{-10} \mathrm{~s}$ for $\mathrm{AG}-2$ to $4.1 \times 10^{3} \mathrm{~s}$ for $\mathrm{AG}-800$. Lifetimes at $E_{\mathrm{T}}+193 \mathrm{~nm}$ passed beyond the normal mass spectrometry time scale (ca. $10^{-5} \mathrm{~s}$ ) at AG-8, a 1042-Da species (based on a curve not shown). Rates also decreased with increasing ion size in the LV model. The rate curves shifted to lower values in the $L V$ model relative to the original model at a given energy (Figure 3). The shift increased as the ions got larger, being more than 10 orders of magnitude for AG-800. This rate reduction was somewhat compensated by increasing $E_{T}$ in the $L V$ model such that at $E_{\mathbf{r}}+193 \mathrm{~nm}$, the rates differ by only about 6 orders of magnitude for $A G-800$. The effect of increasing size given by our two models demonstrates that the decline in dissociation rate with increasing ion

Table 4. Mean frequencies: original versus $L V$ models

\begin{tabular}{ccc}
\hline $\begin{array}{c}\text { Number } \\
\text { of AGs }\end{array}$ & $\begin{array}{c}\bar{\nu} \text { (original) } \\
\left(\mathrm{cm}^{-1}\right)\end{array}$ & $\begin{array}{c}\bar{\nu}(\mathrm{LV}) \\
\left(\mathrm{cm}^{-1}\right)\end{array}$ \\
\hline 2 & 1268.9 & 1263.4 \\
16 & 1222.8 & 1210.3 \\
107 & 1217.0 & 1150.6 \\
401 & 1216.2 & 1041.4 \\
800 & 1215.7 & 949.1 \\
\hline
\end{tabular}

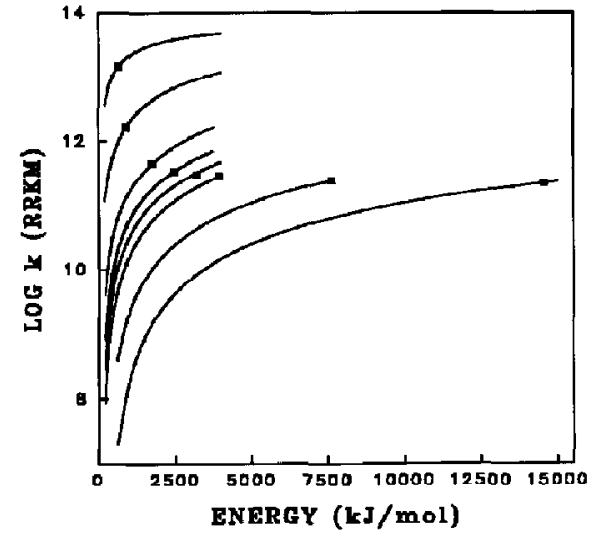

Figure 1. Log(rate constant) ( $k$ in $\left.\mathrm{s}^{-1}\right)$ calculated by the RRKM theory as a function of internal energy for a hypothetical bond cleavage for which $E_{0}=9.65 \mathrm{~kJ} \mathrm{~mol}^{-1}$. For these calculations, frequencies were simply estimated from those for individual structural units (see text). In descending order, the traces are results for the Ala-Gly polymers AG-2 (0.27 kDa), AG-16 (2.07 kDa), AG-65 (8.34 kDa), AG-107 (13.7 kDa), AG-149 (19.1 kDa), $\mathrm{AG}-191(24.5 \mathrm{kDa}), \mathrm{AG}-401(51.3 \mathrm{kDa})$, and AG-800 (102.4 kDa). Squares show $\log k$ at an ion internal energy equal to the thermal energy at $25{ }^{\circ} \mathrm{C}$ plus the energy of a $193-\mathrm{nm}$ photon $(620 \mathrm{~kJ}$ $\mathrm{mol}^{-1}$ ).

size predicted by the RRKM theory does not depend on the set of frequencies utilized in the calculations.

A large ion might absorb more than one photon. Table 5 presents results of calculations using our LV model for absorption of up to 9 photons. According to results in Table 5, absorption of several photons would cause AG-16 to decompose on mass spectrometry time scales, but AG-107 and larger ions would not dissaciate in conventional mass spectrometers even after the absorption of 9 photons. Similar results have been obtained by Schlag and Levine [7].

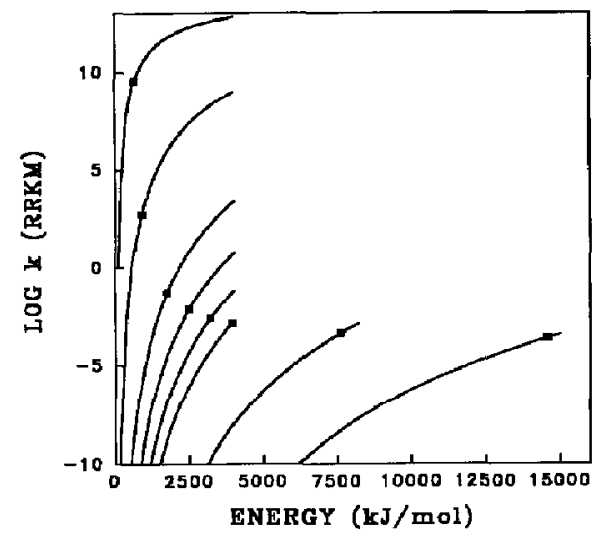

Figure 2. Log(rate constant) as a function of internal energy for a hypothetical bond cleavage for which $E_{0}=100 \mathrm{~kJ} \mathrm{~mol}^{-1}$, using the same frequencies as in Figure 1. The traces are in descending order results for the Ala-Gly polymers AG-2, AG-16, AG-65, AG-107, AG-149, AG-191, AG-401, and AG-800. Squares show $\log k$ at an ion internal energy equal to the thermal energy at 25 ${ }^{\circ} \mathrm{C}$ plus the energy of a $193-\mathrm{nm}$ photon. 


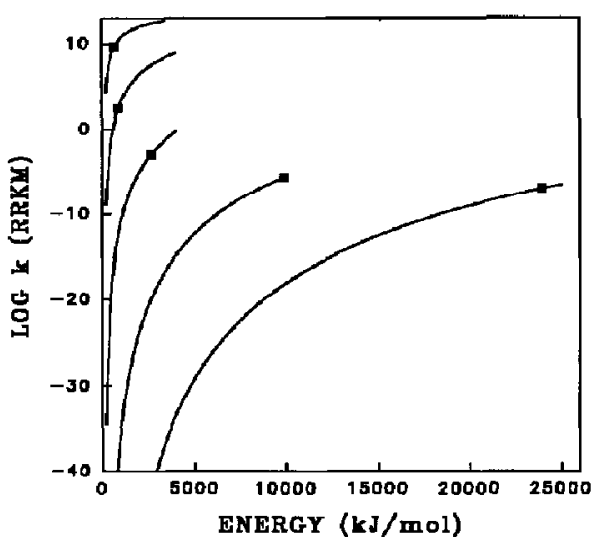

Figure 3. Log(RRKM rate constants) versus internal energy calculated using frequencies lowered with increasing size of the ion to estimate the effects of cooperative modes (see text). Traces in descending order are for AG-2, AG-16, AG-107, AG-401 and AG-800.

Since $E_{0}$ is an important determinant of the rate of bond cleavage, we calculated rate constants using the frequencies of the original model at a series of critical energies for AG-9 and AG-800 at fixed transition state frequencies and an energy content of $E_{\mathrm{T}}$ at $25^{\circ} \mathrm{C}+193$ $\mathrm{nm}$ (Figure 4), A linear relationship was obtained between $\log k$ and $E_{0}$ from $E_{0}=9.6 \mathrm{~kJ} \mathrm{~mol}^{-1}$ to $E_{0}=200$ $\mathrm{kJ} \mathrm{mol}^{-1}$.

\section{Discussion}

According to our RRKM calculations, as ions get larger, their rates of dissociation decrease at a given internal energy. This is in accord with previous experimental and theoretical results [7-11]. Also, as in previous work, the effect of size on the rates is offset somewhat by parallel increases in thermal energy content. However, for larger ions, adding thermal and photon energy still gives rates far too slow to produce decomposition in the mass spectrometer when $E_{0}=100 \mathrm{~kJ}$ $\mathrm{mol}^{-1}$. This contrasts with conclusions from RRK cal- culations at $E_{0}=48 \mathrm{~kJ} \mathrm{~mol}^{-1}$ [8]. Furthermore, dissociation on mass spectrometry time scales is not predicted at $E_{0}=100 \mathrm{~kJ} \mathrm{~mol}^{-1}$ even after absorption of multiple 193-nm photons. Thus, at $E_{0}=100 \mathrm{~kJ}$ $\mathrm{mol}^{-1}$, the calculations suggest that impractically large amounts of energy would have to be added to fragment large ions.

The calculated decomposition rates at $E_{\mathrm{T}}+193 \mathrm{~nm}$ are very high at low $E_{0}$, but decrease rapidly with increasing critical energy (Figure 4). At $E_{0}=9.65 \mathrm{~kJ}$ $\mathrm{mol}^{-1}$, ions of all sizes were predicted to decompose in the ion source even at very low internal energies. $\mathrm{Up}$ to $E_{0}=35 \mathrm{~kJ} \mathrm{~mol}^{-1}$, the calculations predict that significant fractions of all ions would decompose in the ion source or at least before reaching the field-free regions of a conventional mass spectrometer. At critical energies above about $50 \mathrm{~kJ} \mathrm{~mol}{ }^{-1}$ and for jons larger than about $1 \mathrm{kDa}$, the calculations predict difficulty in adding enough energy to produce fragmentations on mass spectrometry time scales $\left(1 \times 10^{-5} \mathrm{~s}\right)$. Since this limit is a strong function of the temperature and critical energy for fragmentation, this correlates reasonably with experimental findings [5] that the upper size limit to obtaining useful fragments on conventional mass spectrometers is about $1500 \mathrm{Da}$. However, decompositions, possibly collision induced, of larger ions have been observed in a time-of-flight mass spectrometer following matrix-assisted laser desorption ionization [21].

Despite the apparently reasonable agreement between the RRKM calculated lifetimes and observed ones, the underlying assumption of the model, that energy flows freely through the ion such that there is a quasi-equilibrium between states constituting the transition state for decomposition and all of the remaining states of the ion, may not hold for very large molecules. This view has recently been taken by Schlag and Levine [7] in addressing issues related to those considered here. To rationalize observed decomposition rates of large ions that are much higher than predicted by RRK theory, they point out that when vibrational excitation is below one quantum per mode, vibrational relaxation

Table 5. Log $k($ RRKM) as a function of thermal energy at $298 \mathrm{~K}$ plus number of 193-nm photons absorbed for model LV Ala-Gly peptides ${ }^{\text {a }}$

\begin{tabular}{|c|c|c|c|c|c|c|}
\hline \multirow{2}{*}{$\begin{array}{c}\text { Number } \\
\text { of photons }\end{array}$} & \multicolumn{2}{|c|}{ AG-16 } & \multicolumn{2}{|c|}{ AG-107 } & \multicolumn{2}{|c|}{$A G-800$} \\
\hline & $E$ & $\log k$ & $\bar{E}$ & $\log k$ & $\bar{E}$ & $\log k$ \\
\hline 0 & 284 & -5.38131 & 2102 & -4.95464 & 23333 & -7.30472 \\
\hline 1 & 898 & 2.55784 & 2636 & -4.00882 & 23948 & -7.01815 \\
\hline 2 & 1512 & 5.21715 & 3250 & -1.56314 & 24562 & -6.74124 \\
\hline 3 & 2126 & 6.70353 & 3864 & -0.4251 & 25176 & -6.47350 \\
\hline 4 & 2740 & 7.68541 & 4478 & 0.50533 & 25790 & -6.21438 \\
\hline 5 & 3355 & 8.39482 & 5092 & 1.28671 & 26464 & -5.96344 \\
\hline 6 & 3969 & 8.93739 & 5707 & 1.95619 & 27019 & -5.72026 \\
\hline 7 & 4583 & 9.3691 & 6321 & 2.53881 & 27635 & -5.48444 \\
\hline 8 & 5197 & 9.72278 & 6935 & 3.05222 & 28247 & -5.25561 \\
\hline 9 & 5811 & 10.01908 & 7549 & 3.50932 & 28861 & -5.03345 \\
\hline
\end{tabular}

${ }^{a} E_{\mathrm{a}}=100 \mathrm{~kJ} \mathrm{~mol}^{-1}$. Units of $E$ are $\mathrm{kJ} \mathrm{mol}^{-1}$. Units of $k$ are $\mathrm{s}^{-1}$. 


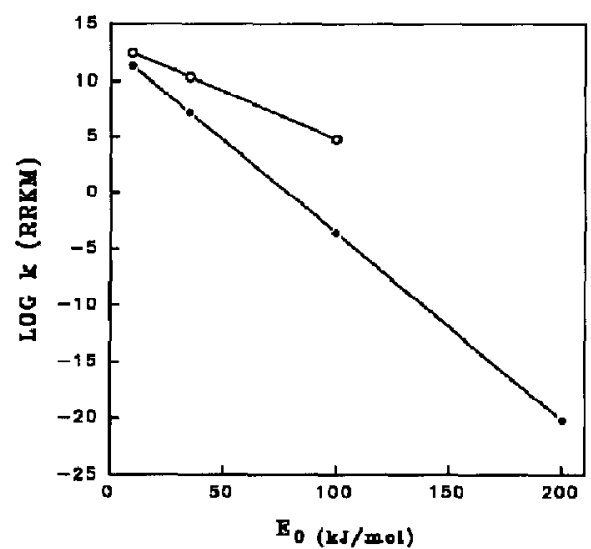

Figure 4. Dependence of the rate constant of a decomposition on the critical energy for that decomposition. The rates are calculated at an internal energy equal to the estimated thermal energy in the neutral precursor at $25^{\circ} \mathrm{C}$ plus the energy added by the absorption of a $193 \mathrm{~nm}$ photon. Open circles, results for an ion containing 9 Ala-Gly units. Filled circles, results for an ion containing 800 Ala-Gly units. Note that the points for each ion lie on a straight line, and that the slope of this line steepens with the size of the ion.

will be much slower than assumed in the RRK-RRKM theories. They suggest that excitation may remain localized in large ions such that the effective number of degrees of freedom is much smaller than the total number in the ion, leading a fraction of the ions to fragment more rapidly than predicted by RRK theory.

Heating would be a way to increase the fragmentation of large ions on mass spectrometry time scales, since the amount of energy added by that means is proportional to ion size, although Schlag and Levine [7] suggest that the effect of thermal energy on reaclion rates is not always significant. However, heating is a useful way to dissociate multiply charged peptide ions produced by electrospray mass spectrometry [22].

Our results suggest that to determine structures of large molecules by mass spectrometry, an alternative to adding larger amounts of energy is needed to generate characteristic fragments. Methods that can be used on relatively simple instruments are also desirable. Cotter, Fenselau, and co-workers [23, 24] recognized these needs, leading them to apply endothermic ion-molecule reactions to generating structure-specific fragmentations of peptide and other biological molecules. Utilizing chemical reactions in lieu of adding more energy seems a promising approach to fragmenting ionized macromolecules in the gas phase. Ideally, such reactions would be mass spectrometric equivalents of the Edman method of sequencing peptides [25] and the Maxam-Gilbert method for sequencing DNA [26], that is, they would produce fragments identifying and locating each unit in the macromolecule. The lower $E_{0}$, the faster the fragmentation, so ion-molecule reactions that reduce $E_{0}$ as much as possible should be sought. An exothermic reaction without an activation energy would be ideal. In such a reaction, bond making with the reagent could be syn- chronized with bond breaking in the ion-for example, an exothermic SN2 reaction-so that the reaction rate would not depend on intramolecular vibrational energy relaxation.

\section{Acknowledgments}

We thank the Robert A. Welch Foundation (grant BD 1106 to LLG) for financial support, Gregory Robak for help with the figures, a referee for many helpful comments, and Ms. Debbie Pavlu for typing. We further thank Alfred Lowrey for providing a set of scaled frequencies from his ab initio optimization of alanylglycine.

\section{References}

1. MacFarlane, R. D. Acc. Chem. Res. 1982, 15, 268-275.

2. Karas, M.; Hillenkamp, F. Anal. Chem. 1988, 60, 2299-2301.

3. Fenn, J. B.; Mann, M.; Meng, C. K.; Wong, S. K; Whitehouse, C. M. Mass Spectrom. Reo. 1990, 9, 37.

4. Barber, M.; Bordoli, R. S.; Elliott, G. J.; Horoch, N. J.; Green, B. N. Biochem. Biophys. Res. Commun. 1983, 110, 753-757.

5. Neumann, R. M.; Sheil, M. M.; Derrick, P. J. Z. Naturforsch. 1984, 39, $584-592$.

6. Demirev, P;Olthoff, J. K; Fenselau, C.; Cotter, R. J. Anal. Chem. 1987, 59, 1951-1954.

7. Schlag, E. W.; Levine, R. D. Chem. Phys. Lett. 1989, 163, $523-530$.

8. McKeown, P. J.; Johnston, M. V. J. Ant. Soc. Mass Spectrom. $1991,2,103-107$.

9. Lin, Y. N,; Rabinovitch, B. S. T. Phys. Chem. 1970, 74, $1769-1775$.

10. Bente, P. F. III; Mclafferty, F. W.; McAdoo, D. J.; Lifshitz, C. J. Phys. Chem. 1975, 79, 713-721.

11. McAdoo, D. J.; Bente, P. F. III; Gross, M. L.; McLafferty, F. W. Org. Mass Spectrom. 1974, 9, 525-535.

12. Forst, $W$. Theory of Unimolecular Reactions; Academic Press: New York, 1973; pp. 14-17.

13. McAdoo, D. J.; Traeger, J. C.; Griffin, L. L.; Hudson, C. E.; Ahmed, M. S.; Giam, C. S. Proceedings of the 39 th Conference on Mass Spectrometry and Allied Topics; Nashville, TN, 1991, 895-896.

14. Hase, W. L.; Bunker, D. L. Quantum Chemistry Program Exchange; Chemistry Department, Indiana University: Bloomington, IN; Program No. 234.

15. Bunker, D. L.; Wang, F.-M. I. Am. Chem. Soc. 1977, 99, 7457-7459.

16. Pulay, P.; Fogarassi, G.; Pang, F; Boggs, J. E. J. Am. Chem. Soc. 1979, 101, 2550-2560.

17. Shimanouchi, T.; Matsuura, H.; Ogawa, Y.; Harada, I. J. Phys. Chem. Ref. Data 1978, 7, 1323-1443.

18. McAdoo, D. J.; Traeger, J. C.; Hudson, C. E.; Griffin, L. L. J. Phys. Chem. 1988, 92, 1524-1530.

19. Rice, O. K. Statistical Mechanics, Thermodyntamics and Kinetics; W. H. Freeman: San Francisco, 1967; pp. 41-46.

20. Williams, E. R.; McLafferty, F. W. J. Am. Soc. Mass Spectrom. 1990, 1, 361-365.

21. Spengler, B.; Kirsch, D.; Kaufmann, R. Rapid Commun. Mass Spectrom. 1991, 5, 198-202.

22. Rockwood, A. L.; Busman, M.; Udseth, H. R.; Smith, R. D. Rapid Commun. Mass Spectrom. 1991, 5, 582-585.

23. Orlando, R.; Murphy, C.; Fenselau, C.; Hansen, G.; Cotter, R. J. Anal. Chem. 1990, 62, 125-129.

24. Orlando, R.; Fenselau, C.; Cotter, R. J. J. Am. Soc. Mass Spectrom. 1991, 2, 189-197.

25. Hunkapiler, M. W.; Hood, L. E. Sciente 1983, 219, 650-659.

26. Maxam, A. M.; Gilbert, W. Proc. Natl. Acad. Sci. 1977, 74, $560-564$. 